\title{
Diffusion with optimal resetting
}

This article has been downloaded from IOPscience. Please scroll down to see the full text article.

2011 J. Phys. A: Math. Theor. 44435001

(http://iopscience.iop.org/1751-8121/44/43/435001)

View the table of contents for this issue, or go to the journal homepage for more

Download details:

IP Address: 129.175.97.14

The article was downloaded on 21/02/2012 at 14:04

Please note that terms and conditions apply. 


\title{
Diffusion with optimal resetting
}

\author{
Martin R Evans $^{1,2}$ and Satya N Majumdar ${ }^{2,3}$ \\ ${ }^{1}$ SUPA, School of Physics and Astronomy, University of Edinburgh, Mayfield Road, \\ Edinburgh EH9 3JZ, UK \\ 2 Department of Physics of Complex Systems, Weizmann Institute of Science, Rehovot 76100, \\ Israel \\ ${ }^{3}$ University Paris-Sud, CNRS, LPTMS, UMR 8626, Orsay F-01405, France \\ E-mail: m.evans@ed.ac.uk and satya.majumdar@u-psud.fr
}

Received 21 July 2011, in final form 19 September 2011

Published 11 October 2011

Online at stacks.iop.org/JPhysA/44/435001

\begin{abstract}
We consider the mean time to absorption by an absorbing target of a diffusive particle with the addition of a process whereby the particle is reset to its initial position with rate $r$. We consider several generalizations of the model of Evans and Majumdar (2011 Phys. Rev. Lett. 106 160601): (i) a space-dependent resetting rate $r(x)$; (ii) resetting to a random position $z$ drawn from a resetting distribution $\mathcal{P}(z)$; and (iii) a spatial distribution for the absorbing $\operatorname{target} P_{T}(x)$. As an example of (i) we show that the introduction of a non-resetting window around the initial position can reduce the mean time to absorption provided that the initial position is sufficiently far from the target. We address the problem of optimal resetting, that is, minimizing the mean time to absorption for a given target distribution. For an exponentially decaying target distribution centred at the origin we show that a transition in the optimal resetting distribution occurs as the target distribution narrows.
\end{abstract}

PACS numbers: 05.40.-a, 02.50.-r, 87.23.Ge

(Some figures may appear in colour only in the online journal)

\section{Introduction}

Search problems occur in a variety of contexts: from animal foraging [1] to the target search of proteins on DNA molecules [2-4]; from internet search algorithms to the more mundane matter of locating one's mislaid possessions. Often search strategies involve a mixture of local steps and long-range moves [5-9] and these are referred to as intermittent search strategies. For human searchers at least, a natural tendency is to return to the starting point of the search after the length of time spent searching becomes excessive.

In a recent paper [10] we modelled such a strategy as a diffusion process with an additional rate of resetting to the starting point $x_{0}$ with rate $r$. Considering the object of the search to be 
an absorbing target at the origin, the duration of the search becomes the time for the diffusing particle to reach the origin. Statistics such as the mean time to absorption of the process then give a measure of the efficiency of the search strategy, defined by the resetting rate $r$. Moreover, the model provides a system where the statistics of absorption times can be computed exactly.

A related model, where searchers have some probabilistic lifetime after which another searcher will be sent out, has been studied by Gelenbe [11] and mean times to absorption computed. Also, a model of an intermittent search where searchers undergo phases of 'teleportation' to random positions has previously been studied [12]. In the mathematical literature, the mean first passage time for random walkers that have the option of restarting at the initial position has been considered [13].

In [10], it was shown that the mean first passage time (MFPT) to the origin for a single diffusive searcher becomes finite in the presence of resetting (in contrast to a purely diffusive search where the MFPT diverges). Moreover the MFPT has a minimum value as a function of the resetting rate $r$ to the fixed initial position $x_{0}$. Thus, there is an optimal resetting rate $r$ as a function of the distance to the target $x_{0}$.

In this work, we address the question of resetting strategies which optimize the MFPT in a wider context. To this end, we make several generalizations of single-particle diffusion with resetting studied in [10]. First, we consider a space-dependent resetting rate $r(x)$. Second, we consider resetting to a random position $z$ (rather than a fixed $x_{0}$ ) drawn from a resetting distribution $\mathcal{P}(z)$. Finally, we consider a probability distribution for the absorbing $\operatorname{target} P_{T}(x)$. The general question we ask is: what are the optimal functions $r(x), \mathcal{P}(x)$ that minimize the MFPT for a given $P_{T}(x)$ ? Although we do not propose a general solution, the examples we study turn up some surprising results and illustrate that answers to the problem may be non-trivial.

The paper is organized as follows. In section 2 we review the calculation of the mean first passage time for one-dimensional diffusion in the presence of resetting to the initial position with rate $r$. In section 3 we introduce spatial dependent resetting $r(x)$ and work out the example of a non-resetting window of width $a$ around the initial point. In section 4 we consider the generalization to a resetting distribution $\mathcal{P}(z)$ and to a distribution of the target site $P_{T}(x)$. In section 5 we formulate the general problem of optimizing the mean first passage time with respect to the resetting distribution $\mathcal{P}(z)$. We consider the example of an exponential target distribution and show that there is a transition in the optimal resetting distribution. We conclude in section 6 .

\section{First passage time for single particle diffusion with resetting}

We begin by briefly reviewing the one-dimensional case of diffusion with resetting to the initial position $x_{0}$ (see figure 1), introduced in [10]. The Master equation for $p\left(x, t \mid x_{0}\right)$, the probability distribution for the particle at time $t$ having started from initial position $x_{0}$, reads

$$
\frac{\partial p\left(x, t \mid x_{0}\right)}{\partial t}=D \frac{\partial^{2} p\left(x, t \mid x_{0}\right)}{\partial x^{2}}-r p\left(x, t \mid x_{0}\right)+r \delta\left(x-x_{0}\right),
$$

with initial condition $p\left(x, 0 \mid x_{0}\right)=\delta\left(x-x_{0}\right)$. In equation (1) $D$ is the diffusion constant of the particle and $r$ is the resetting rate to the initial position $x_{0}$. The second term on the right-hand side (rhs) of equation (1) denotes the loss of probability from the position $x$ due to reset to the initial position $x_{0}$, while the third term denotes the gain of probability at $x_{0}$ due to resetting from all other positions.

The stationary state of (1) is the solution of

$$
D \frac{\partial^{2} p^{*}\left(x \mid x_{0}\right)}{\partial x^{2}}-r p^{*}\left(x \mid x_{0}\right)=-r \delta\left(x-x_{0}\right),
$$




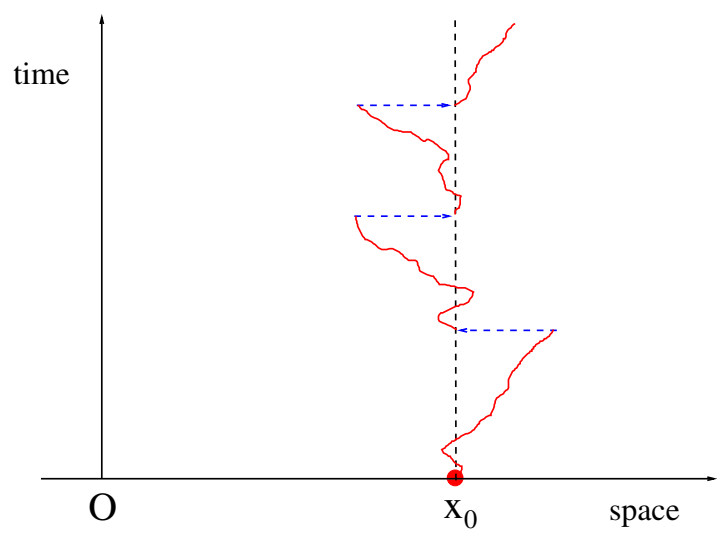

Figure 1. Schematic spacetime trajectory of a one-dimensional Brownian motion that starts at $x_{0}$ and resets stochastically to its initial position $x_{0}$ at rate $r$.

which is determined by the elementary Green function technique, which we now recall.

The solutions to the homogeneous counterpart of (2) are $\mathrm{e}^{ \pm \alpha_{0} x}$ where

$$
\alpha_{0}=\sqrt{r / D}
$$

The solution to (2) is constructed from linear combinations of these solutions which satisfy the following boundary conditions: $p^{*} \rightarrow 0$ as $x \rightarrow \pm \infty$, and $p^{*}$ is continuous at $x=x_{0}$. Imposing these conditions yields

$$
p^{*}\left(x \mid x_{0}\right)=A \exp \left(-\alpha_{0}\left|x-x_{0}\right|\right) .
$$

Note that (4) has a cusp at $x=x_{0}$. The constant $A$ is fixed by the discontinuity of the first derivative at $x=x_{0}$ which is determined by integrating (2) over a small region about $x_{0}$

$$
\left.\frac{\partial p^{*}\left(x \mid x_{0}\right)}{\partial x}\right|_{x \rightarrow x_{0}^{+}}-\left.\frac{\partial p^{*}\left(x \mid x_{0}\right)}{\partial x}\right|_{x \rightarrow x_{0}^{-}}=-\alpha_{0}^{2} .
$$

Carrying this out yields $A=\alpha_{0} / 2$ so that

$$
p^{*}\left(x \mid x_{0}\right)=\frac{\alpha_{0}}{2} \exp \left(-\alpha_{0}\left|x-x_{0}\right|\right) .
$$

Alternatively, the constant $A$ in (4) could be fixed by the normalization of the probability distribution (4).

Note that (6) is a non-equilibrium stationary state by which it is meant that there is circulation of probability even in the one-dimensional geometry. At all points $x$ there is always a diffusive flux of probability in the direction away from $x_{0}$ given by $-D \partial p / \partial x$, and a nonlocal resetting flux in the opposite direction from all points $x \neq x_{0}$ to $x_{0}$.

\subsection{Mean first passage time}

We now consider the mean first passage time for the diffusing particle to reach the origin. One can think of an absorbing target at the origin which instantaneously absorbs the particle (see e.g. [14]).

A standard approach to first-passage problems is to use the backward Master equation where one treats the initial position as a variable (for a review see [15]). Let $Q(x, t)$ denote the survival probability of the particle up to time $t$ (i.e. the probability that the particle has not 
visited the origin up to time $t$ ) starting from the initial position $x$. The boundary and initial conditions are $Q(0, t)=0, Q(x, 0)=1$ (see e.g. [16] for more general reaction boundary conditions).

The backward Master equation (where the variable $x$ is now the initial position) reads for the survival probability $Q(x, t)$

$$
\frac{\partial Q(x, t)}{\partial t}=D \frac{\partial^{2} Q(x, t)}{\partial x^{2}}-r Q(x, t)+r Q\left(x_{0}, t\right) .
$$

Note that $Q(x, t)$ depends implicitly on the resetting position $x_{0}$ due to the third term on the right hand side of (7). The second and third terms on the rhs correspond to the resetting of the initial position from $x$ to $x_{0}$, which implies a loss of probability from $Q(x, t)$ and a gain of probability to $Q\left(x_{0}, t\right)$.

Equation (7) may be derived as follows. We consider the survival probability $Q(x, t+\Delta t)$ up to time $t+\Delta t$, where $\Delta t$ is a small interval of time. We divide the time interval $[0, t+\Delta t]$ into two intervals: $[0, \Delta t]$ and $[t, t+\Delta t]$. In the first interval $[0, \Delta t]$, there are two possibilities: (i) with probability $r \Delta t$, the particle may be reset to $x_{0}$ and then for the subsequent interval $[\Delta t, t+\Delta t]$ this $x_{0}$ will be the new starting position and (ii) with probability $(1-r \Delta t)$, no resetting takes place, but instead the particle diffuses to a new position $(x+\xi)$ in time $\Delta t$, where $\xi$ is a random variable distributed according to a gaussian distribution $P(\xi)=(4 \pi D \Delta t)^{-1 / 2} \exp \left(-\xi^{2} / 4 D \Delta t\right)$. This new position $(x+\xi)$ then becomes the starting position for the subsequent second interval $[\Delta t, t+\Delta t]$. One then sums over all possible values of $\xi$ drawn from $P(\xi)$. Note that we are implicitly using the Markov property of the process whereby for the second interval $[\Delta t, t+\Delta t]$, only the end position of the first interval $[0, \Delta t]$ matters. Taking into account these two possibilities, one then gets

$$
Q(x, t+\Delta t)=r \Delta t Q\left(x_{0}, t\right)+(1-r \Delta t) \int \mathrm{d} \xi P(\xi) Q(x+\xi, t),
$$

which can be rewritten as

$$
\begin{aligned}
\frac{Q(x, t+\Delta t)-Q(x, t)}{\Delta t}=\int \frac{\mathrm{d} \xi}{\Delta t} P(\xi)(Q(x+\xi, t) \\
-Q(x, t))+r Q\left(x_{0}, t\right)-r Q(x, t)+O(\Delta t) .
\end{aligned}
$$

Taking the limit $\Delta t \rightarrow 0$ then yields (7).

The mean first passage time $T$ to the origin beginning from position $x$ is obtained by noting that $-\frac{\partial Q(x, t)}{\partial t} \mathrm{~d} t$ is the probability of absorption by the target in time $t \rightarrow t+\mathrm{d} t$. Therefore, on integrating by parts, we have

$$
T=-\int_{0}^{\infty} t \frac{\partial Q(x, t)}{\partial t} \mathrm{~d} t=\int_{0}^{\infty} Q(x, t) \mathrm{d} t
$$

(assuming that $t Q(x, t) \rightarrow 0$ as $t \rightarrow \infty)$. Integrating (7) with respect to time yields

$$
-1=D \frac{\partial^{2} T(x)}{\partial x^{2}}-r T(x)+r T\left(x_{0}\right)
$$

with boundary conditions $T(0)=0$ and $T(x)$ finite as $x \rightarrow \infty$.

To solve for the mean first passage time beginning at the resetting position $x=x_{0}>0$ we first consider the initial position to be at $x>0$, different from the resetting position $x_{0}$, then solve (11) with arbitrary $x$ and $x_{0}$. Once we have this solution we set $x=x_{0}$ to determine $T\left(x_{0}\right)$ self-consistently.

The general solution to (11) is

$$
T(x)=A \mathrm{e}^{\alpha_{0} x}+B \mathrm{e}^{-\alpha_{0} x}+\frac{1+r T\left(x_{0}\right)}{r},
$$




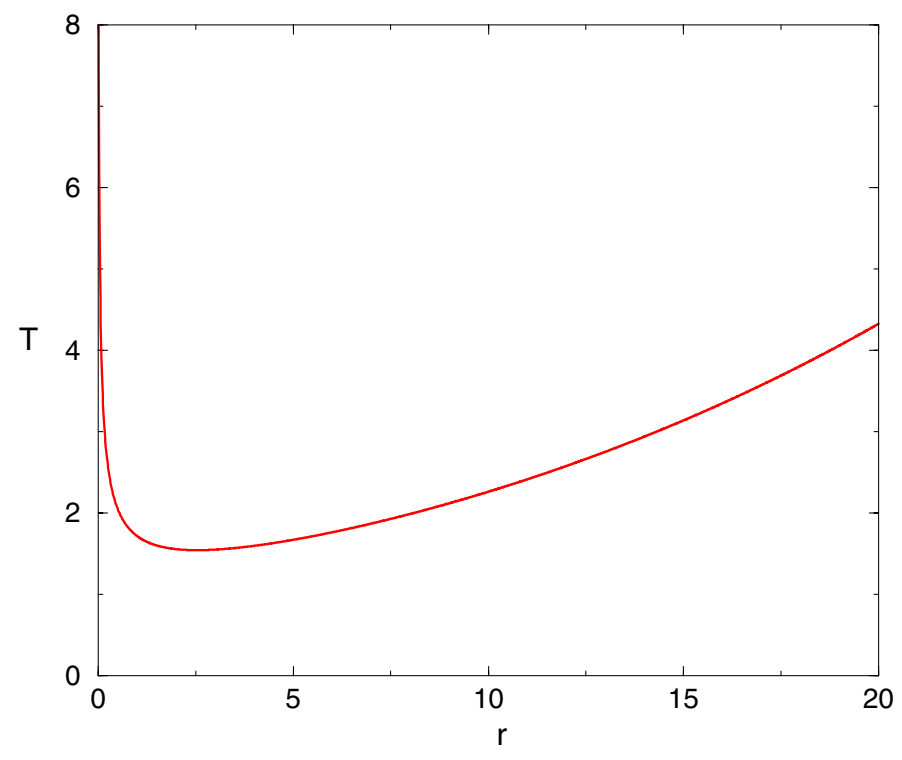

Figure 2. The mean first passage time $T=\frac{1}{r}\left[\exp \left(\sqrt{r / D} x_{0}\right)-1\right]$ plotted as a function of $r$ for fixed $x_{0}=1$ and $D=1$. clearly $T$ diverges as $r \rightarrow 0$ and as $r \rightarrow \infty$ with a single minimum at $r^{*}=2.53962 \ldots$

where $\alpha_{0}=\sqrt{r / D}$. The boundary condition that $T(x)$ is finite as $x \rightarrow \infty$ implies $A=0$ and the boundary condition $T(0)=0$ fixes $B$. Thus,

$$
T(x)=\frac{1+r T\left(x_{0}\right)}{r}\left[1-\mathrm{e}^{-\alpha_{0} x}\right] .
$$

Solving for $T\left(x_{0}\right)$ self-consistently yields

$$
T\left(x_{0}\right)=\frac{1}{r}\left[\exp \left(\alpha_{0} x_{0}\right)-1\right]=\frac{1}{r}\left[\exp \left(\sqrt{r / D} x_{0}\right)-1\right] .
$$

Note from (14) that, for fixed $x_{0}, T$ is finite for $0<r<\infty$. As a function of $r$ for fixed $x_{0}, T$ diverges when $r \rightarrow 0$ as

$$
T \simeq \frac{x_{0}}{(D r)^{1 / 2}}
$$

This is expected since as $r \rightarrow 0$, one should recover the pure diffusive behaviour (no resetting) for which the $T$ is divergent-due to the large excursions that take the diffusing particle away from the target at the origin. Also $T$ diverges rapidly as $r \rightarrow \infty$, the explanation being that as the reset rate increases the diffusing particle has less time between resets to reach the origin. In other words, the high resetting rate to $x_{0}$ cuts off the trajectories that bring the diffusing particle towards the target.

We now consider $T$ as a function of $r$ for a given value of $x_{0}$ and define the reduced variable

$$
z=\alpha_{0} x_{0}=\left(\frac{r}{D}\right)^{1 / 2} x_{0}
$$

Since $T$ diverges as $r \rightarrow 0$ and $r \rightarrow \infty$ it is clear that there must be a minimum of $T$ with respect to $r$ (see figure 2). The condition for the minimum, $\frac{\mathrm{d} T}{\mathrm{~d} r}=0$, reduces to the transcendental equation

$$
\frac{z}{2}=1-\mathrm{e}^{-z}
$$


which has a unique non-zero solution $z^{*}=1.59362 \ldots$ In terms of the resetting rate, this means an optimal resetting rate $r^{*}=\left(z^{*}\right)^{2} D / x_{0}^{2}=(2.53962 \ldots) D / x_{0}^{2}$, for which the mean first passage time $T\left(x_{0}\right)$ is minimum. The dimensionless variable $z(16)$ is a ratio of two lengths: $x_{0}$, the distance from the resetting point to the target, and $(D / r)^{1 / 2}$, which is the typical distance diffused between resetting events. Thus, for fixed $D$ and $x_{0}$ the mean first passage time of the particle can be minimized by choosing $r$ so that this ratio takes the value $z^{*}$.

\section{Space-dependent resetting rate}

In this section, we generalize the model of section 2 to the case of space-dependent resetting rate $r(x)$.

The Master equation for the probability distribution $p\left(x, t \mid x_{0}\right)$ is generalized from (1) to

$$
\frac{\partial p\left(x, t \mid x_{0}\right)}{\partial t}=D \frac{\partial^{2} p\left(x, t \mid x_{0}\right)}{\partial x^{2}}-r(x) p\left(x, t \mid x_{0}\right)+\int \mathrm{d} x^{\prime} r\left(x^{\prime}\right) p\left(x^{\prime}, t \mid x_{0}\right) \delta\left(x-x_{0}\right) \text {. }
$$

The third term on the right-hand side now represents the flux of probability injected at $x_{0}$ through resetting from all points $x \neq x_{0}$.

The stationary distribution $p^{*}\left(x \mid x_{0}\right)$ satisfies

$$
D \frac{\partial^{2} p^{*}\left(x \mid x_{0}\right)}{\partial x^{2}}-r(x) p^{*}\left(x \mid x_{0}\right)=-\int \mathrm{d} x^{\prime} r\left(x^{\prime}\right) p^{*}\left(x^{\prime} \mid x_{0}\right) \delta\left(x-x_{0}\right) .
$$

In general, the stationary state is difficult to determine unless $r(x)$ has some simple form. The equation for the mean first passage time becomes

$$
-1=D \frac{\partial^{2} T(x)}{\partial x^{2}}-r(x) T(x)+r(x) T\left(x_{0}\right) .
$$

Again, this is difficult to solve generally for arbitrary $r(x)$.

In the following we consider a solvable example where $r(x)$ is zero in a window around $x_{0}$ and is constant outside this window.

\subsection{Example of a non-resetting window}

We consider the case of a non-resetting window of width $a$ about $x_{0}$, within which the resetting process does not occur:

$$
\begin{aligned}
r(x) & =0 \text { for }\left|x-x_{0}\right|<a \\
& =r \text { for }\left|x-x_{0}\right| \geqslant a .
\end{aligned}
$$

This choice is a rather natural one in the sense that a typical searcher usually does not reset when it is close to its starting point, but rather the resetting event occurs when it diffuses a certain threshold distance $a$ away from its initial position.

The Master equation reads

$$
\begin{array}{rlrl}
\frac{\partial p\left(x, t \mid x_{0}\right)}{\partial t} & =D \frac{\partial^{2} p\left(x, t \mid x_{0}\right)}{\partial x^{2}}+r h(t) \delta\left(x-x_{0}\right) & \left|x-x_{0}\right|<a \\
& =D \frac{\partial^{2} p\left(x, t \mid x_{0}\right)}{\partial x^{2}}-r p\left(x, t \mid x_{0}\right) \quad\left|x-x_{0}\right| \geqslant a,
\end{array}
$$

where

$$
h(t)=\int \mathrm{d} x p\left(x, t \mid x_{0}\right) \theta\left(\left|x-x_{0}\right|-a\right)
$$




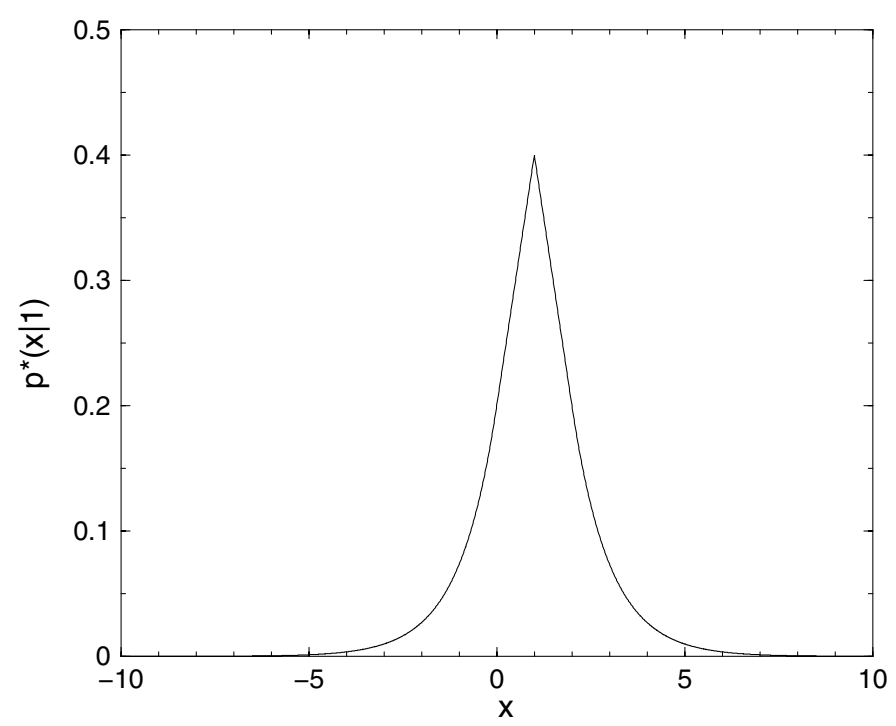

Figure 3. The stationary solution $p^{*}\left(x \mid x_{0}\right)$ in equation (26)-(27) plotted as a function of $x$, for the choice $x_{0}=1, a=1, r=1$ and $D=1$. The non-resetting window is over $x \in[0,2]$ with the initial position at $x_{0}=1$. The solution is symmetric around $x_{0}=1$ with a cusp at $x=x_{0}=1$.

with initial condition $p(x, 0)=\delta\left(x-x_{0}\right)$. Thus $h(t)$ is the probability that the particle is outside the non-resetting window, i.e. in the resetting zone at time $t$; the particle is reset to the origin with a total rate $h(t) r$.

First, we consider the stationary state. One can solve for the stationary probability using the Green function technique of section 1. For $\left|x-x_{0}\right|>a$ (outside the window), $p^{*}\left(x \mid x_{0}\right)$ satisfies $D \frac{\partial^{2} p^{*}\left(x \mid x_{0}\right)}{\partial x^{2}}=r p^{*}\left(x \mid x_{0}\right)$ and should tend to zero as $|x| \rightarrow \infty$. For $0<\left|x-x_{0}\right|<a$ (inside the window), $p^{*}\left(x \mid x_{0}\right)$ satisfies $D \frac{\partial^{2} p^{*}\left(x \mid x_{0}\right)}{\partial x^{2}}=0$ for all $x \neq x_{0}$. The solution should be continuous at $x=x_{0}$, but its derivative must undergo a jump at $x=x_{0}$ and the jump discontinuity can be computed by integrating equation (23) across $x=x_{0}$.

Thus, noting that the solution should be symmetric about $x=x_{0}$, one has

$$
\begin{aligned}
p^{*}\left(x \mid x_{0}\right) & =A \exp \left(-\alpha_{0}\left(\left|x-x_{0}\right|-a\right)\right) \quad \text { for } \quad\left|x-x_{0}\right|>a \\
& =A-B\left(\left|x-x_{0}\right|-a\right) \quad \text { for } \quad\left|x-x_{0}\right|<a,
\end{aligned}
$$

where $\alpha_{0}=\sqrt{r / D}$ and the constants $A$ and $B$ are determined by the discontinuity in the derivative of $p^{*}\left(x \mid x_{0}\right)$ at $x=x_{0}$ and the continuity of the derivative at $\left|x-x_{0}\right|=a$.

The result is

$$
A=\frac{\alpha_{0}^{2}}{2+2 a \alpha_{0}+a^{2} \alpha_{0}^{2}}, \quad B=\alpha_{0} A
$$

The solution has a cusp at $x=x_{0}$ and a discontinuity in the second derivative at $\left|x-x_{0}\right|=a$ (see figure 3).

We now consider an absorbing trap at the origin. The backward equation for $T(x)$, the mean time to absorption beginning from $x$, reads

$$
-1=D \frac{\partial^{2} T(x)}{\partial x^{2}}-r T(x)+r T\left(x_{0}\right) \quad \text { for } \quad\left|x-x_{0}\right|>a
$$




$$
-1=D \frac{\partial^{2} T(x)}{\partial x^{2}} \quad \text { for } \quad\left|x-x_{0}\right|<a .
$$

The general solution to (30) is

$$
T(x)=A x+B-\frac{x^{2}}{2 D}
$$

and the solution to (29) that does not diverge as $x \rightarrow \infty$ is

$$
\begin{array}{ll}
T(x)=\frac{1+r T\left(x_{0}\right)}{r}+C \mathrm{e}^{-\alpha_{0}\left(x-x_{0}-a\right)} & \text { for } \quad x>x_{0}+a \\
T(x)=\frac{1+r T\left(x_{0}\right)}{r}+E \mathrm{e}^{-\alpha_{0} x}+F \mathrm{e}^{\alpha_{0} x} & \text { for } \quad x<x_{0}-a .
\end{array}
$$

The constants $A, B, C, E, F$ are determined by the continuity of $T(x)$ and $T^{\prime}(x)$ at $\left|x-x_{0}\right|=a$ and the boundary condition $T(0)=0$. The result for $T\left(x_{0}\right)$ is

$$
\begin{gathered}
T\left(x_{0}\right)=\frac{1}{r\left(1+a \alpha_{0}\right)}\left[\cosh \alpha_{0}\left(x_{0}-a\right)\left(1+2 a \alpha_{0}+\frac{3 a^{2} \alpha_{0}^{2}}{2}+a^{3} \alpha_{0}^{3}\right)\right. \\
\left.+\sinh \alpha_{0}\left(x_{0}-a\right)\left(1+2 a \alpha_{0}+\frac{3 a^{2} \alpha_{0}^{2}}{2}\right)\right]-\frac{1}{r} .
\end{gathered}
$$

We now consider the reduced parameters $z=\alpha_{0} x_{0}$ and $y=\alpha_{0} a$, and $T$ as a function $y$ for $z$ fixed. The allowed values of $y$ are $0 \leqslant y \leqslant z$. At $y=z$, one can show that $\left.\frac{\mathrm{d} T}{\mathrm{~d} y}\right|_{y=z}>0$. Therefore the minimum of $T$ with respect to $y$ is either at $y=0$ or at a non trivial minimum $0 \leqslant y \leqslant z$.

The condition for a minimum $\frac{\mathrm{d} T}{\mathrm{~d} y}=0$ reduces to

$$
\frac{2+y}{4+5 y+2 y^{2}}=\tanh (z-y) \text {. }
$$

Therefore the condition for there to be a nontrivial minimum for $y>0$ is given by $\tanh z>1 / 2$ or equivalently $z>(\log 3) / 2=0.5493 \ldots$

In summary, the analysis of the condition for $T(y)$ to be a minimum reveals that: if $z<(\log 3) / 2$ then $y=0$ is the minimum of $T(y)$; if $z>(\log 3) / 2$ then $T(y)$ has a nontrivial minimum at $0<y<z$. Therefore, when $z<(\log 3) / 2$ the introduction of a window around the initial site where resetting does not take place does not reduce the mean time to absorption. A strategy of introducing a non-resetting window is an effective one only when the initial point is sufficiently far from the search target; otherwise it is advantageous to always reset.

\subsection{Optimal resetting function}

Having seen in the previous example that non-trivial behaviour emerges for a simple spatialdependent resetting rate $r(x)$, one can ask for the optimal function $r(x)$. The optimization problem would be to minimize $T$ under certain constraints pertaining to the information available to the searcher. Clearly if there are no constraints, that is one can use full information about the target position, the optimal strategy is to reset immediately whenever $x>x_{0}$ and not reset when $x<x_{0}$. This corresponds to the choice

$$
\begin{array}{lll}
r(x)=0 & \text { for } & x<x_{0} \\
r(x)=\infty & \text { for } & x>x_{0} .
\end{array}
$$

In this case problem (20) reduces to the mean first passage time of a diffusive particle with reflecting barrier at $x_{0}$ the solution of which is

$$
T^{*}\left(x_{0}\right)=\frac{x_{0}^{2}}{2 D}
$$


Thus, (36) gives the lowest possible mean first passage time for a diffusive process. One can then ask about how close simple strategies, such as a spatially constant resetting rate $r$ or non-resetting window, come to approaching this bound.

For example, the case of spatially constant resetting rate $r$ considered in section 2 yields a minimum MFPT using (17)

$$
T=\frac{x_{0}^{2}}{D} \frac{\left(\mathrm{e}^{z}-1\right)}{z^{2}}=\frac{x_{0}^{2}}{2 D} \frac{\mathrm{e}^{z^{*}}}{z^{*}}=3.0883 \ldots T^{*}\left(x_{0}\right)
$$

As noted in section 3.1, the value 3.0883 may be improved upon by considering a non-resetting window around $x_{0}$.

However, (36) uses the crucial information of whether the target (at $x=0$ ) is to the right or left of the resetting site $x_{0}$. More realistically, the searcher would not have this information. The relevant optimization problem is to find the optimal resetting rate $r\left(\left|x-x_{0}\right|\right)$ (constrained to be a function of the distance $\left|x-x_{0}\right|$ from the resetting site) that minimizes $T\left(x_{0}\right)$. This remains an open problem.

\section{Resetting distribution and target distribution}

In this section, we consider the generalization to a system with resetting to points distributed according to $\mathcal{P}(z)$. We shall also consider a distribution of the target site $P_{T}(x)$.

\subsection{Stationary state}

We begin by considering again the one-dimensional case of diffusion but this time with resetting to a random position: at rate $r$ the particle is reset to a random position $z \rightarrow z+\mathrm{d} z$ drawn with probability $\mathcal{P}(z) \mathrm{d} z$. We refer to $\mathcal{P}(z)$ as the reset distribution. For simplicity we take the initial position $x_{0}$ to be distributed according to the same distribution as the reset position $p\left(x_{0}, 0\right)=\mathcal{P}\left(x_{0}\right)$.

The Master equation for the probability density $p(x, t)$ now reads

$$
\frac{\partial p(x, t)}{\partial t}=D \frac{\partial^{2} p(x, t)}{\partial x^{2}}-r p(x, t)+r \mathcal{P}(x) \text {. }
$$

The stationary solution to (38) is simply found using (6) as the Green function:

$$
p^{*}(x)=\int \mathrm{d} z \mathcal{P}(z) p^{*}(x \mid z),
$$

which, using $p^{*}\left(x \mid x_{0}\right)$ given by (6), yields

$$
\left.p^{*}(x)=\frac{\alpha_{0}}{2} \int \mathrm{d} z \mathcal{P}(z) \exp \left(-\alpha_{0}|x-z|\right)\right) .
$$

\subsection{Mean first passage time}

The mean first passage time, $T\left(x_{0}, x_{T}\right)$, to a target point $x_{T}$, starting from $x_{0}$ with resetting distribution $\mathcal{P}(z)$, satisfies

$$
-1=D \frac{\partial^{2} T\left(x_{0}, x_{T}\right)}{\partial x_{0}^{2}}-r T\left(x_{0}, x_{T}\right)+r \int \mathrm{d} z \mathcal{P}(z) T\left(z, x_{T}\right),
$$

with boundary condition $T\left(x_{T}, x_{T}\right)=0$. To solve this equation we let

$$
F\left(x_{T}\right)=\int \mathrm{d} z \mathcal{P}(z) T\left(z, x_{T}\right),
$$

then write down the general solution to (41) and solve for $F\left(x_{T}\right)$ self-consistently. 
The general solution of (41) which is finite as $x_{0} \rightarrow \infty$ is

$$
T\left(x_{0}, x_{T}\right)=A \mathrm{e}^{-\alpha_{0}\left|x_{0}-x_{T}\right|}+\frac{1}{r}+F\left(x_{T}\right) .
$$

The boundary condition $T\left(x_{T}, x_{T}\right)=0$ implies $A=-\left(\frac{1}{r}+F\right)$. Then substituting this expression for $A$ in (43) and integrating we find

$$
F\left(x_{T}\right)=\left(\frac{1}{r}+F\left(x_{T}\right)\right)\left(1-\int \mathrm{d} z \mathcal{P}(z) \mathrm{e}^{-\alpha_{0}\left|z-x_{T}\right|}\right),
$$

which yields

$$
F\left(x_{T}\right)=\frac{1}{r}\left(\frac{\alpha_{0}}{2 p^{*}\left(x_{T}\right)}-1\right) .
$$

Inserting this into (43) we obtain

$$
T\left(x_{0}, x_{T}\right)=\frac{\alpha_{0}}{2 r p^{*}\left(x_{T}\right)}\left[1-\exp \left(-\alpha_{0}\left|x_{0}-x_{T}\right|\right)\right] .
$$

As noted above it is convenient to choose the same distribution for $x_{0}$ as the resetting distribution. Averaging over $x_{0}$ then gives using (39)

$$
T\left(x_{T}\right)=\frac{1}{r}\left[\frac{\alpha_{0}}{2 p^{*}\left(x_{T}\right)}-1\right] .
$$

Equation (47) gives the expression for the mean first passage time to a target positioned at $x_{T}$. Let us check the case of a single position $x_{0}$ to which the particle is reset $\mathcal{P}(z)=\delta\left(z-x_{0}\right)$. In this case (47) becomes

$$
T\left(x_{T}\right)=\frac{1}{r}\left[\exp \left(\alpha_{0}\left|x_{T}-x_{0}\right|\right)-1\right],
$$

which recovers (14) when $x_{T}$ is set to 0 .

Finally, we average over possible target positions drawn from a target distribution: $P_{T}\left(x_{T}\right)$

$$
\bar{T}=\frac{1}{r}\left[\frac{\alpha_{0}}{2} \int \mathrm{d} x_{T} \frac{P_{T}\left(x_{T}\right)}{p^{*}\left(x_{T}\right)}-1\right] .
$$

Equation (49) gives the main result of this section-the MFPT for a resetting distribution $\mathcal{P}\left(x_{0}\right)$ and averaged over target distribution $P_{T}\left(x_{T}\right)$.

\section{Extremization of mean first passage time}

Let us now consider the problem of extremizing $\bar{T}$ given by (49), for a given target distribution $P_{T}(x)$, with respect to the resetting distribution $\mathcal{P}(z)$. Throughout this section we will assume a symmetric target distribution: $P_{T}(x)=P_{T}(-x)$ and $P_{T}^{\prime}(x)=-P_{T}^{\prime}(-x)$.

The problem is to minimize the functional appearing in (49): $\int \mathrm{d} x \frac{P_{T}(x)}{p^{*}(x)}$ where

$$
p^{*}(x)=\frac{\alpha_{0}}{2} \int \mathrm{d} z \mathcal{P}(z) \mathrm{e}^{-\alpha_{0}|z-x|}
$$

subject to the constraint $\int \mathrm{d} z \mathcal{P}(z)=1$. The functional derivative to be satisfied is

$$
\frac{\delta}{\delta \mathcal{P}(y)}\left[\int \mathrm{d} x \frac{P_{T}(x)}{p^{*}(x)}+\lambda \int \mathrm{d} x \mathcal{P}(x)\right]=0
$$

where $\lambda$ is a Lagrange multiplier. Condition (51) yields

$$
\int \mathrm{d} x \frac{P_{T}(x)}{\left[p^{*}(x)\right]^{2}} \mathrm{e}^{-\alpha_{0}|y-x|}=\frac{2 \lambda}{\alpha_{0}} .
$$


For (52) to hold for all $y$ requires that

$$
\frac{P_{T}(x)}{\left[p^{*}(x)\right]^{2}}=\lambda,
$$

or fixing $\lambda$ through the normalization of $p^{*}(x)$

$$
p^{*}(x)=\frac{P_{T}^{1 / 2}(x)}{\int \mathrm{d} z P_{T}^{1 / 2}(z)} .
$$

Equation (54) implies that to minimize $T$ the stationary probability distribution should be proportional to the square root of the target distribution. This result has been derived in [17] for the case of searching for the target by sampling a probability distribution $\mathcal{P}(x)$. This corresponds to the limit $r \rightarrow \infty$ of our model. For $r<\infty$ we have the additional constraint that the optimal $p^{*}(x)$ should be realizable from a resetting distribution $\mathcal{P}(z)$ through formula (50).

Equation (50) may be solved for $\mathcal{P}(z)$ for a desired $p^{*}(x)$ by taking the Fourier transform and using the convolution theorem to give

$$
\widetilde{\mathcal{P}}(k)=\left(1+\frac{k^{2}}{\alpha_{0}^{2}}\right) \widetilde{p}^{*}(k),
$$

where $\widetilde{\mathcal{P}}(k)$ is the Fourier transform of $\mathcal{P}(x)$ and $\widetilde{p}^{*}(k)$ is the Fourier transform of $p^{*}(x)$.

We may invert the Fourier transformation to find

$$
\mathcal{P}(x)=p^{*}(x)-\frac{1}{\alpha_{0}^{2}} \frac{\mathrm{d}^{2} p^{*}(x)}{\mathrm{d} x^{2}} .
$$

However, this solution may become negative in which case the solution to the optimization problem is unphysical.

\subsection{Example of an exponential target distribution}

As a simple example, we consider an exponentially decaying target distribution peaked at $x=0$ :

$$
P_{T}(x)=\frac{\beta}{2} \mathrm{e}^{-\beta|x|} .
$$

We first note that for a delta function resetting distribution $\mathcal{P}(z)=\delta\left(z-x_{0}\right)$ the mean first passage time (49) diverges when $\alpha_{0}>\beta$. Therefore, for small $\beta$ (a broad target distribution) one expects an optimal resetting distribution (for fixed $\alpha_{0}$ ) that differs from a delta function.

For $\beta<2 \alpha_{0}$, the optimal stationary distribution is from (54)

$$
p^{*}(x)=\frac{\beta}{4} \mathrm{e}^{-\beta|x| / 2} .
$$

This expression yields from (56) a resetting distribution that is always positive, thus the optimal resetting distribution is

$$
\mathcal{P}(z)=\frac{\beta}{4} \mathrm{e}^{-\beta|z| / 2}\left[1-\frac{\beta^{2}}{4 \alpha_{0}^{2}}\right]+\frac{\beta^{2}}{4 \alpha_{0}^{2}} \delta(z) .
$$

For $\beta>2 \alpha_{0}$, (59) always gives negative probabilities due to the first term. Therefore we anticipate that $\mathcal{P}(x)=\delta(x)$ is at least a locally optimal solution. In fact, one can prove this is the case by showing that any distribution of the form $\mathcal{P}(x)=(1-\epsilon) \delta(x)+\epsilon f(x)$, where $f(x) \geqslant 0$ and $\int \mathrm{d} x f(x)=1$ leads to an increase in (49) at first order in $\epsilon$ when $\beta>2 \alpha_{0}$. (As the proof is straightforward but somewhat tedious we did not include it here.) Thus a transition in the form of the optimal resetting distribution, from a single delta function to (59), occurs at $\beta=2 \alpha_{0}$. 


\subsection{Inversion of $p^{*}(x)$}

As noted above, the constraint $\mathcal{P}(x) \geqslant 0$ means that the optimal $p^{*}(x)$ given by (54) may not be realizable from a physical resetting distribution $\mathcal{P}(z)$. We are therefore led to the general question of when a desired stationary distribution (e.g. (54)) which we denote $g(x)$ may be generated from (50) i.e. when can we invert

$$
g(x)=\frac{\alpha_{0}}{2} \int \mathrm{d} z \mathcal{P}(z) \mathrm{e}^{-\alpha_{0}|z-x|}
$$

to obtain a physical $\mathcal{P}(z)$ ?

Let us first discuss a sufficient condition for the resetting distribution implied by (60) to be physical.

Equation (55) relates the characteristic functions of the two distributions $\mathcal{P}(x)$ and $g(x)$ (given there by $p^{*}(x)$ ). In terms of the characteristic function, Polya's theorem [18] states that if a function $\phi(k)$ satisfies: $\phi(0)=1 ; \phi(k)$ is even; $\phi(k)$ is convex for $k>0$, and $\phi(\infty)=0$; then $\phi(k)$ is the characteristic function of an absolutely continuous symmetric distribution. Polya's theorem therefore gives a sufficient condition for $\mathcal{P}(x)$ implied by $p^{*}(x)$ to be physical.

The condition for convexity becomes in one dimension

$$
\frac{\mathrm{d}^{2}}{\mathrm{~d} k^{2}}\left[\left(1+\frac{k^{2}}{\alpha_{0}^{2}}\right) g(k)\right] \geqslant 0 \text { for all } k \geqslant 0 .
$$

If the function $\widetilde{\mathcal{P}}(k)$ does not satisfy the conditions of Polya's theorem, the solution of (60) is invalid as a probability distribution, i.e. the desired $g(x)$ cannot be realized from any resetting probability distribution $\mathcal{P}(z)$.

In the case where (60) may not be inverted to give a physical $\mathcal{P}(x)$, it may be possible to generate the desired form for $g(x)$ on a finite region by choosing a compact support for $\mathcal{P}(z)$. Let us assume $g(x)$ to be a symmetric function of $x$. Then if we choose

$$
\begin{aligned}
\mathcal{P}(z) & =\lambda\left[g(z)-\frac{1}{\alpha_{0}^{2}} \frac{\mathrm{d}^{2} g(z)}{\mathrm{d} z^{2}}\right] \text { for }|z| \leqslant y_{0} \\
& =0 \text { for }|z|>y_{0},
\end{aligned}
$$

where $\lambda$ is a normalizing constant, we find

$$
\begin{aligned}
& p^{*}(x)=\lambda g(x) \text { for }|x| \leqslant y_{0} \\
& p^{*}(x)=\lambda g\left(y_{0}\right) \mathrm{e}^{\alpha_{0}\left(y_{0}-|x|\right)} \text { for }|x| \geqslant y_{0}
\end{aligned}
$$

provided that $y_{0}$ is chosen so that

$$
\begin{aligned}
& g\left(y_{0}\right)+\frac{1}{\alpha_{0}} g^{\prime}\left(y_{0}\right)=0 \\
& g\left(-y_{0}\right)-\frac{1}{\alpha_{0}} g^{\prime}\left(-y_{0}\right)=0 .
\end{aligned}
$$

(see the appendix). The second condition follows from the first by the assumed symmetry of $g(x)$. As an example, we consider the Gaussian distribution

$$
g(x)=\left(\frac{\beta}{\pi}\right)^{1 / 2} \mathrm{e}^{-\beta x^{2}}
$$

The inversion of (60) using (56) yields

$$
\mathcal{P}(x)=g(x)-\frac{1}{\alpha_{0}^{2}} \frac{\mathrm{d}^{2} g(x)}{\mathrm{d} x^{2}}=\left(\frac{\beta}{\pi}\right)^{1 / 2} \mathrm{e}^{-\beta x^{2}}\left[1+\frac{2 \beta}{\alpha_{0}^{2}}-\frac{4 \beta^{2} x^{2}}{\alpha_{0}^{2}}\right]
$$


which becomes negative for

$$
|x|>\frac{\alpha_{0}}{2 \beta}\left(1+\frac{2 \beta}{\alpha_{0}^{2}}\right)^{1 / 2} .
$$

However, choosing a compact support for $\mathcal{P}(z)$ according to (66) and (67), yields

$$
y_{0}=\frac{\alpha_{0}}{2 \beta}
$$

and we find that the resulting distribution (70) is positive for all $x$.

\section{Conclusion}

In this paper, we have considered some generalizations of diffusion with stochastic resetting to the case of spatial-dependent resetting rate and a resetting distribution. We have considered the mean first passage time to a target which may be situated at a fixed point (the origin) or distributed according to a distribution and derived the result (49). The minimization of this quantity may then be formulated as an optimization problem of which we have studied some simple examples.

In particular, we have seen some perhaps unexpected results. First, the introduction of a non-resetting window around a fixed resetting position reduces the MFPT when the target is sufficiently far away. This suggests that the optimal resetting distribution, in the case where we consider a resetting rate that is symmetric about the resetting point, $r\left(\left|x-x_{0}\right|\right)$ may be non-trivial. We have also seen that in the case of an exponentially distributed target (57) the optimal resetting distribution undergoes a transition from (59) to a pure delta function at the origin.

Generally, the computation of an optimal resetting distribution is an open problem since the resetting distribution that minimizes $\bar{T}$ may be become negative over some domain and therefore nonphysical. In the case where (56) becomes unphysical, although we do not have a solution to the extremization problem of minimizing $\bar{T}$ subject to the additional constraint $\mathcal{P}(x) \geqslant 0$ we may propose likely candidates for extremal solutions. One possibility for the optimal physical solution is one that has compact support i.e. since the constraint for the distribution to be physical is that $\mathcal{P}(x) \geqslant 0$, one might expect that the optimal solution lies on the boundary where $\mathcal{P}(x)=0$ for some regions of $x$. However, we have no proof that this is the case.

Further considerations for optimizing mean first passage times in a more realistic search process would be to add a cost to resetting since in the present model the diffusive particle instantaneously resets to its selected resetting position. This could be implemented by attributing some time penalty to each resetting event, as is the case in the framework intermittent searching.

\section{Appendix. Proof that (63) yields (65)}

We wish to show that expression (63) for $\mathcal{P}(x)$

$$
\begin{aligned}
\mathcal{P}(z) & =\lambda\left[g(z)-\frac{1}{\alpha_{0}^{2}} \frac{\mathrm{d}^{2} g(z)}{\mathrm{d} z^{2}}\right] \text { for }|z| \leqslant y_{0} \\
& =0 \text { otherwise, }
\end{aligned}
$$

yields (64)-(65) for the stationary distribution given by (40), provided that (66)-(67) holds. 
We begin by inserting (A.1)-(A.2) into (40) in the case $|x|<y_{0}$ :

$p^{*}(x)=\frac{\alpha_{0} \lambda}{2}\left\{\int_{-y_{0}}^{x}\left[g(z)-\frac{1}{\alpha_{0}^{2}} \frac{\mathrm{d}^{2} g(z)}{\mathrm{d} z^{2}}\right] \mathrm{e}^{-\alpha_{0}(x-z)}+\int_{x}^{y_{0}}\left[g(z)-\frac{1}{\alpha_{0}^{2}} \frac{\mathrm{d}^{2} g(z)}{\mathrm{d} z^{2}}\right] \mathrm{e}^{-\alpha_{0}(z-x)}\right\}$.

We use the following integration by parts, valid for all $\alpha_{0} \neq 0$

$$
\begin{aligned}
\int_{a}^{b}\left[g(z)-\frac{1}{\alpha_{0}^{2}}\right. & \left.\frac{\mathrm{d}^{2} g(z)}{\mathrm{d} z^{2}}\right] \mathrm{e}^{\alpha_{0} z} \mathrm{~d} z \\
& =\left[g(b)-\left.\frac{1}{\alpha_{0}} \frac{\mathrm{d} g(z)}{\mathrm{d} z}\right|_{z=b}\right] \frac{\mathrm{e}^{\alpha_{0} b}}{\alpha_{0}}-\left[g(a)-\left.\frac{1}{\alpha_{0}} \frac{\mathrm{d} g(z)}{\mathrm{d} z}\right|_{z=a}\right] \frac{\mathrm{e}^{\alpha_{0} a}}{\alpha_{0}} .
\end{aligned}
$$

Inserting this into (A.3) and cancelling terms yields

$$
\begin{aligned}
p^{*}(x)=\frac{\alpha_{0} \lambda}{2}\{ & \frac{2 g(x)}{\alpha_{0}}+\frac{\mathrm{e}^{-\alpha_{0}\left(x+y_{0}\right)}}{\alpha_{0}}\left[g\left(-y_{0}\right)-\left.\frac{1}{\alpha_{0}} \frac{\mathrm{d} g(z)}{\mathrm{d} z}\right|_{z=-y_{0}}\right] \\
& \left.+\frac{\mathrm{e}^{\alpha_{0}\left(x-y_{0}\right)}}{\alpha_{0}}\left[-g\left(y_{0}\right)-\left.\frac{1}{\alpha_{0}} \frac{\mathrm{d} g(z)}{\mathrm{d} z}\right|_{z=y_{0}}\right]\right\} .
\end{aligned}
$$

Then conditions (66)-(67) ensure that $p^{*}(x)=\lambda g(x)$ for $|x|<y_{0}$.

In the case $x>y_{0}$ we find

$$
\begin{aligned}
p^{*}(x) & =\frac{\alpha_{0} \lambda}{2} \int_{-y_{0}}^{y_{0}}\left[g(z)-\frac{1}{\alpha_{0}^{2}} \frac{\mathrm{d}^{2} g(z)}{\mathrm{d} z^{2}}\right] \mathrm{e}^{-\alpha_{0}(x-z)} \mathrm{d} z \\
& =\frac{\alpha_{0} \lambda}{2} \mathrm{e}^{-\alpha_{0} x}\left\{\frac{\mathrm{e}^{\alpha_{0} y_{0}}}{\alpha_{0}}\left[g\left(y_{0}\right)-\left.\frac{1}{\alpha_{0}} \frac{\mathrm{d} g(z)}{\mathrm{d} z}\right|_{z=y_{0}}\right]-\frac{\mathrm{e}^{-\alpha_{0} y_{0}}}{\alpha_{0}}\left[g\left(-y_{0}\right)-\left.\frac{1}{\alpha_{0}} \frac{\mathrm{d} g(z)}{\mathrm{d} z}\right|_{z=-y_{0}}\right]\right\} \\
& =\lambda \mathrm{e}^{-\alpha_{0}\left(x-y_{0}\right)} g\left(y_{0}\right)
\end{aligned}
$$

where conditions (66)-(67) have been used.

Similarly in the case $x<-y_{0}$ we obtain $p^{*}(x)=\lambda g\left(-y_{0}\right) \mathrm{e}^{\alpha_{0}\left(y_{0}+x\right)}$.

\section{Acknowledgment}

MRE and SNM thank the Weizmann Institute for Weston Visiting Professorships.

\section{References}

[1] Bell W J 1991 Searching Behaviour (London: Chapman and Hall)

[2] Berg O G, Winter R B and von Hippel P H 1981 Diffusion-driven mechanisms of protein translocation on nucleic acids: I. Models and theory Biochemistry 20 6929-48

[3] Coppey M, Bénichou O, Voituriez R and Moreau M 2004 Kinetics of target site localization of a protein on DNA: a stochastic approach Biophys. J. 87 1640-9

[4] Bénichou O, Kafri Y, Sheinman M and Voituriez R 2009 Searching fast for a target on DNA without falling to traps Phys. Rev. Lett. 103138102

[5] Bénichou O, Coppey M, Moreau M, Suet P-H and Voituriez R 2005 Optimal search strategies for hidden targets Phys. Rev. Lett. 94198101

[6] Lomholt M A, Koren T, Metzler R and Klafter J 2008 Lévy strategies in intermittent search processes are advantageous Proc. Natl Acad. Sci. 10511055

[7] Bartumeus F and Catalan J 2009 Optimal search behavior and classic foraging theory J. Phys. A: Math. Theor. 42434002

[8] Oshanin G, Lindenberg K, Wio H S and Burlatsky S 2009 Efficient search by optimized intermittent random walks J. Phys. A: Math. Theor. 42434008 
[9] Rojo F, Revelli J, Budd C E, Wio H S, Oshanin G and Lindenberg K 2010 Intermittent search strategies revisited: effect of the jump length and biased motion J. Phys. A: Math. Theor. 43345001

[10] Evans M R and Majumdar S N 2011 Diffusion with stochastic resetting Phys. Rev. Lett. 106160601

[11] Gelenbe E 2010 Search in unknown environments Phys. Rev. E 82061112

[12] Bénichou O, Moreau M, Suet P-H and Voituriez R 2007 Intermittent search process and teleportation J. Chem. Phys. 126234109

[13] Janson S and Peres Y 2010 Hitting times for random walks with restarts arXiv: 1005.4275

[14] Redner S 2001 A Guide to First-Passage Processes (Cambridge: Cambridge University Press)

[15] Majumdar S N 2005 Brownian functionals in physics and computer science Curr. Sci. 892076

[16] Sano H and Tachiya M 1979 Partially diffusion-controlled recombination J. Chem. Phys. 711276

[17] Snider J 2011 Optimal random search for a single hidden target Phys. Rev. E 83011105

[18] Lukacs E 1970 Characteristic Functions (London: Griffin) 